\title{
A Note on the Core of a Monetary Economy
}

\author{
Eric O’N. Fisher*
}

Department of Economics, The Ohio State University, 410 Arps Hall, 1945 North High Street, Columbus, Ohio 43210

This note shows that there are monetary equilibria in the model of overlapping generations that are in the core. Some equilibria have positive stocks of outside money in every generation. These equilibria are thus self-enforcing, and introducing money into an economy need not be tantamount to contriving a new social institution designed to enforce sequential contracts.

\section{INTRODUCTION}

Let's return to Shell's [16] Gedankenexperiment in which George Washington, Spiro Agnew, and Buck Rogers are haggling over chocolates in an all souls' market. Imagine that Washington, Agnew, and Rogers take advantage of their serendipitous meeting and decide to hammer out a constitution describing the profile of the national debt. What would they agree on? Since they enjoy trading, they would likely restrict their attention to allocations that can be supported by Walrasian equilibria. Being enlightened souls, they might also seek a good faith profile of debt that implements Pareto optimal allocations. But here's the rub. Who gets the chocolate wrapper? Who gets the seigniorage in the creation of the national debt? George Washington is a statesman, but history has shown that Spiro Agnew will not give up without a fight. And Buck Rogers probably knows more monetary theory than both of them put together! By characterizing policies that support allocations in the core, this note describes the profiles of national debt that they would assent to. These

* I thank Gerry Garvey for the conversations on the road to the Goodradigbee that sparked my interest in this topic. I also am grateful to an anonymous referee whose comments helped greatly to sharpen the focus of this note. 
policies have the added attraction that even if the Ayatollah reconvened the All Souls' Convention, then neither he nor his followers could propose a credible repudiation of the debt. Thus these plans are bona fide and time-consistent.

In the simplest versions of models of overlapping generations, positive stocks of an outside asset may support Walrasian equilibrium allocations that are not in the core. ${ }^{1}$ The (loose) intuition is that agents in later generations have an incentive to renege on any generational debt they have inherited and then issue their own inside money as an asset for future generations. But this note describes economies in which every generation's temptation to renege on the stock of past debt is weaker than its gains from trade in each period. It extends the results of Esteban and Millan [10] to economies in which the present value of the stock of debt is not constant. Those authors show that Walrasian equilibria with constant positive stocks of outside assets cannot support equilibria in the core, and they conclude that Gale [13] was correct in emphasizing that introducing money into a Walrasian system was analogous to creating a new social institution. This note shows that such a conclusion may be true for many large economies, but it is not uniformly true for economies with only a few agents in each generation or for those where the gains from trade are especially high.

\section{NOTATION AND DEFINITIONS}

The index set of agents is $H=\bigcup_{t=0}^{T} H_{t}$, where $H_{t}$ is the set of agents belonging to generation $t$. I allow $T$ to be finite or infinite. Each agent $h \in H-H_{0}$ lives for two periods, ${ }^{2}$ but agent $h \in H_{0}$ lives for only one period. In each period $t \geqslant 1$, there are $\ell$ commodities that cannot be stored. Hence the endowment of agent $h \in H_{t}$, is $\omega_{h}=\left(0, \ldots, \omega_{h}^{t}, \omega_{h}^{t+1}, 0, \ldots\right)$, with $\omega_{h}^{t} \in \mathfrak{R}_{+}^{\ell}$, and the consumption bundle of agent $h \in H_{t}$, is $x_{h}=\left(0, \ldots, x_{h}^{t}\right.$, $\left.x_{h}^{t+1}, 0, \ldots\right)$, where again $x_{h}^{t} \in \mathfrak{R}_{+}^{\ell}$. The commodity space is $X=\mathfrak{R}_{+}^{\ell} \times$ $\mathfrak{R}_{+}^{\ell} \times \cdots$. The preferences of agent $h \in H_{t}$, are summarized by $u_{h}: \mathfrak{R}_{+}^{2 \ell} \rightarrow \mathfrak{R}$ whose rule is $u_{h}\left(x_{h}\right)=u_{h}\left(x_{h}^{t}, x_{h}^{t+1}\right) .^{3}$

${ }^{1}$ Hendricks et al. [14] showed that there are Walrasian equilibrium that are not in the core. Using a model with two agents per generation, Kovenock [15] showed that there are Pareto optimal Walrasian equilibrium that are not in the (empty) core. Esteban [8] described stationary debt policies that supported allocations in the core, and Esteban [9] gives an excellent survey of this literature.

${ }^{2}$ This assumption is not at all as restrictive as it may seem. Balasko et al. [2] give an algorithm for converting an arbitrary demographic structure into the one described here.

${ }^{3}$ Note that $h \in H_{0}$ is endowed with and consumes commodities only in the (first and) last period of her life and has a utility function $u_{h}: \mathfrak{R}_{+}^{\ell} \rightarrow \mathfrak{R}$ whose rule is $u_{h}\left(x_{h}\right)=u_{h}\left(x_{h}^{1}\right)$. 
Agent $h \in H_{t}$ receives lump-sum transfers of a fiat asset $m_{h}=\left(0, \ldots, m_{h}^{t}\right.$, $\left.m_{h}^{t+1}, 0, \ldots\right)$, with $m_{h}^{t} \in \mathfrak{R}$. If $m_{h}^{t}<0$, then $h$ is taxed at time $t$. This agent's gross additions to money holdings are $y_{h}=\left(0, \ldots, y_{h}^{t}, y_{h}^{t+1}, 0, \ldots\right)$, with $y_{h}^{t} \in \mathfrak{R}$. ${ }^{4}$ Then $y_{h}^{t}-m_{h}^{t}>0$ if and only if $h \in H_{t}$, saves during the first period of her life since she is committing to inventory more money than she receives in transfers. ${ }^{5}$

Let $p^{t} \in \mathfrak{R}_{+}^{\ell}$ be the vector of commodity prices at time $t$. The sequence of such prices is $p=\left(p^{1}, p^{2}, \ldots\right)$, where $p^{t, i}$ is the present price of commodity $(t, i)$ and the normalization is $p^{1,1}=1$. Now let $q^{t} \in \mathfrak{R}_{+}$be the price of the fiat asset at time $t$; then $q=\left(q^{1}, q^{2}, \ldots\right)$ is the sequence of present prices of the asset.

Taking $p$ and $q$ as given, agent $h \in H_{t}$ chooses $x_{h}$ and $y_{h}$ to maximize

$$
u_{h}\left(x_{h}\right)
$$

subject to

(i) $p \cdot x_{h}+q \cdot y_{h} \leqslant p \cdot \omega_{h}+q \cdot m_{h}$;

(ii) $\quad x_{h}^{t} \geqslant 0, \quad x_{h}^{t+1} \geqslant 0$;

and

$$
y_{h}^{t}+y_{h}^{t+1} \geqslant 0
$$

Let $Y_{h}=\left(0, \ldots, y_{h}^{t}, y_{h}^{t}+y_{h}^{t+1}, 0, \ldots\right)$ be the cumulative asset holdings of agent $h \in H_{t}$. Consider also $M^{t}=\sum_{i=1}^{t} \sum_{h \in H_{i-1} \cup H_{i}} m_{h}^{i}$, the sum of all injections of the fiat asset that have occurred up to time $t$. Then $M=$ $\left(M^{1}, M^{2}, \ldots\right)$ is the profile of the stock of the fiat asset.

A perfect-foresight equilibrium is a sequence of goods and asset prices and a corresponding list of equilibrium allocations and asset demands,

$$
\left\{\left(p^{t}, q^{t}\right)\right\}_{t=1}^{\infty} \quad \text { and } \quad\left\{\left(x_{h}, y_{h}\right)\right\}_{h \in H}
$$

such that

(i) $\left(x_{h}, y_{h}\right)$ solves (1);

(ii) $\sum_{h \in H} x_{h} \leqslant \sum_{h \in H} \omega_{h}$;

\footnotetext{
${ }^{4}$ Agent $h \in H_{0}$ receives tax-transfers only in the last period of her life, and it is implicit that $y_{h}^{0}=0$.

${ }^{5}$ This description of savings and borrowing follows Balasko and Shell [4, Section 2] exactly.
} 


$$
\sum_{h \in H} Y_{h} \leqslant M .^{6}
$$

In a perfect foresight equilibrium, the present price of the fiat asset is a constant; hence, $q=\left(q^{0}, q^{0}, \ldots\right)$ for some $q^{0} \in \mathfrak{R}_{+}$. Thus for $h \in H_{t}$ the real effects of lump-sum tax-transfers depend only on the sum $\mu_{h}=m_{h}^{t}+m_{h}^{t+1}$ because $q \cdot m_{h}=q^{0}\left(m_{h}^{t}+m_{h}^{t+1}\right)=q^{0} \mu_{h}$. Then $\mu=\left(\mu_{h}\right)_{h \in H}$ is the government's generational policy. ${ }^{7}$ Let $w_{h}=p \cdot \omega_{h}+q^{0} \mu_{h}$ be the present value of an agent's income, $x_{h}=f_{h}\left(p, w_{h}\right)$ be the solution to (1), $r=\sum_{h \in H} \omega_{h}$ be the stream of resources, and $w=\left(w_{h}\right)_{h \in H}$ be the sequence of incomes. Then $(p, w)$ is a price-income equilibrium associated with resources $r$ if and only if $\sum_{h \in H} f_{h}\left(p, w_{h}\right)=r$. The generational policy $\mu=\left(\mu_{h}\right)_{h \in H}$ is consistent with the price-income equilibrium $(p, w)$ if and only if $w_{h}=p \cdot \omega_{h}+q^{0} \mu_{h}$ for all $h \in H$.

A monetary equilibrium is a perfect foresight equilibrium in which $q^{0}>0$ and $\mu \neq 0$. A monetary economy is a four-tuple $E=\left\langle H, \omega=\left(\omega_{h}\right)_{h \in H}, \mu=\right.$ $\left.\left(\mu_{h}\right)_{h \in H}, u=\left(u_{h}\right)_{h \in H}\right\rangle$ that has a monetary equilibrium. Let $E(k)$ be the $k$-fold replication of $E$, consisting of $k$ identical agents for each $h \in H$. This definition includes replicating the endowment and tax-transfers of each agent in the original economy.

A coalition is a non-empty subset of $H$. For any coalition $S$ and assignment $x=\left(x_{h}\right)_{h \in H}$,

$$
\begin{aligned}
F_{S}(x)= & \left\{r \in X: \sum_{h \in S} z_{h}=r \text { for some assignment } z\right. \text { such that } \\
& \left.u_{h}\left(z_{h}\right) \geqslant u_{h}\left(x_{h}\right) \text { for all } h \in S, \text { with strict inequality for some } h \in S\right\}
\end{aligned}
$$

is the set of resources that allow these agents to improve upon $x$. For a coalition $S$, it will be convenient to use the notation $\omega_{S}=\sum_{h \in S} \omega_{h}$ and $x_{S}=\sum_{h \in S} x_{h}$. Then a coalition $V$ is relevant to another coalition $S$ if for any feasible assignment $x=\left(x_{h}\right)_{h \in H}$ there exists some $a>0$ such that $a \omega_{V}+x_{S} \in F_{S}(x)$. A coalition $S$ is irreducible if, for any two coalitions $U$ and $V$ forming a partition of $S, U$ is relevant to $V$. Finally, a feasible assignment $x=\left(x_{h}\right)_{h \in H}$ belongs to the core if $\omega_{S} \notin F_{S}(x)$ for any coalition $S$.

${ }^{6}$ A referee pointed out that (1) has an indeterminate solution for money holdings since any $y_{h}$ such that $y_{h}^{t}+y_{h}^{t+1}=0$ will satisfy this program. This observation is true, but there is only one such $y_{h}$ that is consistent with equilibrium since (iii) takes as given the pattern of taxes and transfers. Thus utility maximization determines both $y_{h}^{t}-m_{h}^{t}$ and $y_{h}^{t+1}-m_{h}^{t+1}$. It is convenient to think of (ii) as an equilibrium in the flows of commodities and (iii) as an equilibrium in the end-ofperiod stocks of money.

${ }^{7}$ This apt terminology is that of Auerbach et al. [1]. 


\section{THE CORE OF A MONETARY ECONOMY}

At prices $p=\left(p^{1}, p^{2}, \ldots\right)$ with $p^{t} \in \mathfrak{R}_{++}^{\ell}$ for all $t \geqslant 1$, the expenditure necessary to achieve utility no less that $\bar{u}_{h}$ is $e_{h}\left(p, \bar{u}_{h}\right)=\min \left\{p \cdot x_{h} \mid u_{h}\left(x_{h}\right) \geqslant\right.$ $\left.\bar{u}_{h}\right\}$. I shall impose henceforth that each agent's utility function is continuous and represents preferences that are locally nonsatiated, and I will also consider only equilibria in which every present price is strictly positive. In this case, $e_{h}\left(p, \bar{u}_{h}\right)$ is continuous and increasing in $\bar{u}_{h}$. Consider now such a price sequence $p$ and a fixed stream of endowments $\omega=\left(\omega_{h}\right)_{h \in H}$. For every coalition $S$ define $B(p, S, \omega)$ as the difference between the value of the coalition's endowments and the maximal value of the consumption allocations it can provide its members, both evaluated at prices $p$. Thus

$$
B(p, S, \omega) \equiv \min _{x_{S} \leqslant \omega_{S}}\left\{p \cdot \omega_{S}-\sum_{h \in S} e_{h}\left(p, u_{h}\left(x_{h}\right)\right)\right\} .
$$

Three properties of $B(p, S, \omega)$ are noteworthy. First, this function is defined for all finite coalitions $S$. Second, if $\bar{x}=\left(\bar{x}_{h}\right)_{h \in H}$ is supported by $p$ and $\bar{x}_{S}=\omega_{S}$ for some coalition $S$, then $B(p, S, \omega)=0$. Thus a coalition that is self sufficient has no gains from trade at prices $p$. Finally, since $p \cdot \bar{x}_{h} \leqslant$ $p \cdot \omega_{h}$ in an economy without money, $B(p, S, \omega)$ is the value of market access that $S$ achieves from trading with $H-S$ at prices $p .{ }^{8}$

Now consider explicitly the generational accounts $\mu=\left(\mu_{h}\right)_{h \in H}$ for the monetary economy $E=\left\langle H, \omega=\left(\omega_{h}\right)_{h \in H}, \mu=\left(\mu_{h}\right)_{h \in H}, u=\left(u_{h}\right)_{h \in H}\right\rangle$. I consider first the case where $\# H<\infty$.

Proposition 1. Assume that there are only finitely many agents in $E$, and consider a price income equilibrium $(p, w)$. Then the equilibrium allocations are in the core if and only if $\mu_{H}=0$ and

$$
-B(p, S, \omega) \leqslant q^{0} \mu_{S} \text { for any coalition } S .
$$

Proof. Note first that Balasko and Shell [5, Proposition 3.2] establish that $\mu_{H}=\sum_{h \in H} \mu_{h}=0$ is necessary for $\mu=\left(\mu_{h}\right)_{h \in H}$ to be consistent with $(p, w)$.

Let $\bar{x}=\left(\bar{x}_{h}\right)_{h \in H}$ be the equilibrium allocations, and assume that $-B(p, S, \omega) \leqslant q^{0} \mu_{S}$ for any coalition $S$. Then, for any non-empty $S \subset H, \quad \min _{x_{S} \leqslant \omega_{S}}\left\{p \cdot \omega_{S}-\sum_{h \in S} e_{h}\left(p, u_{h}\left(x_{h}\right)\right)\right\} \geqslant-q^{0} \mu_{S}$. Hence, $\max _{x_{S} \leqslant \omega_{S}}\left\{\sum_{h \in S} e_{h}\left(p, u_{h}\left(x_{h}\right)\right)\right\} \leqslant p \cdot \omega_{S}+q^{0} \mu_{S}=p \cdot \bar{x}_{S}$, and $\omega_{S} \notin F_{S}(\bar{x})$. Thus the equilibrium allocations are in the core.

${ }^{8}$ For a finite coalition $S, B(p, S, \omega) / p \cdot \omega_{S}$ is akin to Debreu's [7] definition of economic loss for an economy with production. 
Now assume that $\bar{x}=\left(\bar{x}_{h}\right)_{h \in H}$ are in the core. Then at goods prices $p$ and asset price $q^{0}, \max _{x_{S} \leqslant \omega_{S}}\left\{\sum_{h \in S} e_{h}\left(p, u_{h}\left(x_{h}\right)\right)\right\} \leqslant p \cdot \bar{x}_{S}=p \cdot \omega_{S}+q^{0} \mu_{S}$ for any non-empty $S \subset H$. Hence, $\min _{x_{S} \leqslant \omega_{S}}\left\{p \cdot \omega_{S}-\sum_{h \in S} e_{h}\left(p, u_{h}\left(x_{h}\right)\right)\right\}=$ $B(p, S, \omega) \geqslant-q^{0} \mu_{S}$.

Q.E.D

The $2^{\# H}-1$ inequalities and the one equality in Proposition 1 describe completely the set of generational policies that are consistent with $(p, w)$ and support allocations in the core of $E$. This set is closed and not empty since it contains the policy $\mu_{h}=0$ for all $h \in H$. Of course, the core of $E$ is not empty because it contains the Walrasian equilibrium allocations. Also, the set of generational policies consistent with $(p, w)$ and supporting allocations in the core will typically have non-empty interior. Only if $B(p, S, \omega)=0$ for every $S \subset H$ will this set consist of the singleton $\mu_{h}=0$ for all $h \in H$. Thus there are non-trivial generational policies that support allocations in the core as long as at least one agent experiences strictly positive gains from trade in a given Walrasian equilibrium.

Equation (3) states simply that no coalition can be taxed more than the value of its gains from trade. The practical interpretation of (3) is problematic because it entails constraints both on the profile of generational policies through time and the distribution of taxes across agents in the same cohort. Proposition 1 serves as a strong reminder that the distribution of taxes and transfers within each cohort matters in determining which policies support allocations in the core. With that proviso firmly in mind, I shall postulate that $\# H_{t}=1$ in the rest of this note. This assumption allows a simple characterization of the dynamic profile of generational policies.

In an economy with infinitely many agents, two problems crop up immediately. First, a coalition may consist of subsets of agents who have no feasible trades. Second, (2) may not be well defined for a coalition with infinitely many members. But (2) is well defined for all coalitions $S$ such that $\# S<\infty$ or $\#(H-S)<\infty$. In an economy with one agent per cohort, there is only one type of irreducible coalition with infinitely many members. Let $S^{t}=\bigcup_{i=0}^{t} H_{i}$; then only infinite coalitions of the form $H-S^{t}$ might improve upon a proposed allocation. Then we can consider some $\left(\tilde{x}_{h}\right)_{h \in H-S^{t}}$ that minimizes (2) for $H-S^{t} .{ }^{9}$ Since this coalition is self-sufficient from $t+2$ onward, $B\left(p, H-S^{t}, \omega\right)=p^{t+1} \cdot\left(\omega_{H_{t+1}}^{t+1}-\tilde{x}_{H_{t+1}}^{t+1}\right)$, which is different from zero only if there are at least two goods per period.

In an economy with only one good per period, any generation being taxed on net can block the equilibrium allocations by forming a coalition

\footnotetext{
${ }^{9}$ Assume that resources are uniformly bounded, and recall that $e_{h}\left(p, u_{h},\left(x_{h}\right)\right)$ is a continuous function of $x_{h}$. Then (2) defines $\left(\tilde{x}_{h}\right)_{h \in H-S^{t}}$ as an argument that minimizes a continuous bounded non-negative function on a domain that is compact in the product topology; hence these allocations exist for any coalition $H-S^{t}$.
} 
consisting of its members only. But in an economy with at least two goods per period, such a coalition may not improve upon these allocations if trade between the generations is sufficiently important to outweigh the taxes needed to service the debt. Proposition 2 formalizes this intuition.

Proposition 2. Assume that aggregate resources are uniformly bounded, and consider a price income equilibrium $(p, w)$ such that $\lim _{i \rightarrow \infty}\left\|p^{t}\right\|$ $=0$. Then the equilibrium allocations are in the core if and only if $\lim \inf _{t \rightarrow \infty} \sum_{i=0}^{t} \mu_{H_{i}}=0$, and

$$
-B\left(p, S^{t}, \omega\right) \leqslant q^{0} \mu_{s^{t}} \leqslant B\left(p, H-s^{t}, \omega\right) \quad \text { for all } t \geqslant 0 .
$$

Proof. Now Balasko and Shell [4, Proposition 5.5] show that $\lim \inf _{t \rightarrow \infty} \sum_{i=0}^{t} \mu_{H_{i}}=0$ is necessary for $\mu=\left(\mu_{h}\right)_{h \in H}$ to be consistent with $(p, w)$. Also, the equilibrium allocations are Pareto optimal since aggregate resources are uniformly bounded. Further, we need consider only irreducible coalitions since any other coalition cannot improve upon the equilibrium allocations.

Let $\bar{x}=\left(\bar{x}_{h}\right)_{h \in H}$ be these allocations, and put $\mu_{S^{t}}=\sum_{i=0}^{t} \mu_{H_{i}}$. If $-B\left(p, S^{t}, \omega\right) \leqslant q^{0} \mu_{S^{t}} \quad$ then $\max _{x_{S^{t}} \leqslant \omega_{S^{t}}}\left\{\sum_{h \in S^{t}} e_{h}\left(p, u_{h}\left(x_{h}\right)\right)\right\} \leqslant p \cdot \omega_{S^{t}}+$ $q^{0} \mu_{S^{t}}=p \cdot \bar{x}_{S^{t}}$, and $\omega_{S^{t}} \notin F_{S^{t}}(\bar{x})$. Market clearing implies that $p \cdot\left(\omega_{H-S^{t}}-\bar{x}_{H-S^{t}}\right)=q^{0} \mu_{S^{t}}$. Hence, if $q^{0} \mu_{S^{t}} \leqslant B\left(p, H-S^{t}, \omega\right)$, then $p \cdot\left(\omega_{H-S^{t}}-\bar{x}_{H-S^{t}}\right) \leqslant \min _{x_{H-S^{t}} \leqslant \omega_{H-S^{t}}}\left\{p \cdot \omega_{H-S^{t}}-\sum_{h \in H-S^{t}} e_{h}\left(p, u_{h}\left(x_{h}\right)\right)\right\}$, and $\omega_{H-S^{t}} \notin F_{H-S^{t}}(\bar{x})$. Finally, the grand coalition cannot improve upon $\bar{x}=\left(\bar{x}_{h}\right)_{h \in H}$ since these allocations are Pareto optimal; thus they are in the core.

Assume now that $\bar{x}=\left(\bar{x}_{h}\right)_{h \in H}$ are in the core. Then at goods prices $p$ and asset price $q^{0}, \max _{x_{S^{t}} \leqslant \omega_{S^{t}}}\left\{\sum_{h \in S^{t}} e_{h}\left(p, u_{h}\left(x_{h}\right)\right)\right\} \leqslant p \cdot \bar{x}_{S^{t}}=p \cdot \omega_{S^{t}}+q^{0} \mu_{S^{t}}$ for any finite $t$. Hence, $\min _{x_{S^{t}} \leqslant \omega_{S^{t}}}\left\{p \cdot \omega_{S^{t}}-\sum_{h \in S^{t}} e_{h}\left(p, u_{h}\left(x_{h}\right)\right)\right\}=$ $B\left(p, S^{t}, \omega\right) \geqslant-q^{0} \mu_{S^{t}}$ for all finite coalitions $S^{t}$. Also, since $q^{0} \mu_{S^{t}}=$ $p \cdot\left(\omega_{H-S^{t}}-\bar{x}_{H-S^{t}}\right)$ is the present value of resources transferred

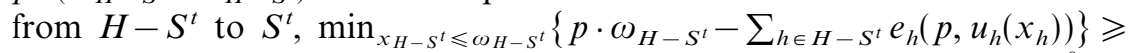
$p \cdot\left(\omega_{H-S^{t}}-\bar{x}_{H-S^{t}}\right)$. But this inequality implies that $B\left(p, H-S^{t}, \omega\right) \geqslant q^{0} \mu_{S^{t}}$.

Q.E.D

In Proposition 1, it was necessary to assume that $\mu_{H}=0$. If there are finitely many agents in the economy, then $\mu_{S}=-\mu_{H-S}$ and (3) implies immediately that $-B(p, S, \omega) \leqslant \mu_{S} \leqslant B(p, H-S, \omega)$. Thus (3) can be restated in a manner entirely analogous to (4), and it captures precisely the intuition that generational debt is a transfer of resources between two groups. In the case of an economy with one agent per cohort, (4) describes completely the set of generational polices that support allocations in the core. 
Equation (4) generalizes Esteban's [8, Proposition 3] characterization of the core of a monetary economy in two directions. First, it shows that the assumption of weakly separable preferences is not necessary for a simple characterization of policies supporting allocations in the core. Second, it allows for non-stationary policies. Since $B\left(p, H-S^{t}, \omega\right) \leqslant p^{t+1} \cdot \omega_{H_{t+1}}^{t+1}$ and resources are uniformly bounded, it is obvious that positive amounts of generational debt must eventually be retired. But this requirement is also imposed by the assumption that $\mu=\left(\mu_{h}\right)_{h \in H}$ is consistent with a priceincome equilibrium in Esteban and Millan's [10] classical set, a generalization of Gale's [12] notion of a classical equilibrium.

In neither of these propositions was it necessary to make strong assumptions about preferences or endowments. Both propositions assumed the existence of an equilibrium with a valued fiat asset, and I was able to use the assumption of existence to characterize fully the set of generational policies that supported equilibrium allocations in the core. Still, the economy in Proposition 2 was small in the extreme, since only two agents trade in each period. Of course, it is interesting to see whether there are large economies with positive stocks of money in every period that support allocations in the core.

Proposition 3. There are monetary economies $E$ satisfying $\mu_{S^{t}}=$ $\sum_{i=0}^{t} \mu_{H_{i}}>0$ for all $t \geqslant 0$ that support equilibrium allocations in the core for every replication $E(k)$.

Proof. I will give an example of such an economy.

Let $\# H_{t}=1$ for all $t \geqslant 0$, and set $\ell=2$. Let $\omega_{h}=((2,1),(0,0), \ldots)$ if $h \in H_{0}$ and $\omega_{h}=((0,0), \ldots,(0,1),(2,1),(0,0), \ldots)$ if $h \in H-H_{0}$. Let preferences be summarized by

$$
u\left(x_{h}\right)=\left\{\begin{array}{l}
\log \left(\min \left\{x_{h}^{1,1}, x_{h}^{1,2}\right\}\right) \\
\quad \text { if } h \in H_{0} \\
\log \left(\min \left\{x_{h}^{t, 1}, x_{h}^{t, 2}\right\}\right)+\log \left(\min \left\{x_{h}^{t+1,1}, x_{h}^{t+1,2}\right\}\right) \\
\quad \text { if } h \in H_{t}, \text { with } t \geqslant 1 .
\end{array}\right.
$$

Since there is only one agent per generation, I can write $\mu=\left(\mu_{h}\right)_{h \in H}=$ $\left(\mu_{t}\right)_{t=0}^{\infty}$ without confusion. Let $\mu=(1,-3 / 4,-3 / 16, \ldots)$ and fix $0<q^{0}<1 / 5$. Then a perfect foresight equilibrium is supported by $p^{t, 1}=p^{t, 2}=$ $\left(3-15 q^{0}\right)(1 / 3)^{t}+20 q^{0}(1 / 4)^{t}$. The equilibrium allocations satisfy $x_{h}^{1,1}=$ $x_{h}^{1,2}=\left(3+q^{0}\right) / 2 \quad$ if $h \in H_{0}$. Also, $\quad x_{h}^{t, 1}=x_{h}^{t, 2}=\left(1-(1 / 4)^{t-1} q^{0}\right) / 2$ and $x_{h}^{t+1,1}=x_{h}^{t+1,2}=\left(3+(1 / 4)^{t} q^{0}\right) / 2$ if $h \in H_{t}$ with $t \geqslant 1$.

Consider $E(k)$, the $k$ th replication of this economy. No coalition of the form $H-S^{t-1}$ can improve upon the equilibrium allocations because it is endowed with none of commodity $(t, 1)$. Assume now that some coalition 
including $k_{1}$ agents in $H_{t-1}$ and $k_{2}$ agents in $H_{t}$ can improve upon these equilibrium allocations. Then endowments are such that the system of equations

$$
\begin{array}{r}
4 k_{1}>k_{1}\left(3+(1 / 4)^{t-1} q^{0}\right)+k_{2}\left(1-(1 / 4)^{t-1} q^{0}\right) \\
2\left(k_{1}+k_{2}\right)>k_{1}\left(3+(1 / 4)^{t-1} q^{0}\right)+k_{2}\left(1-(1 / 4)^{t-1} q^{0}\right)
\end{array}
$$

must be true. These two equations are equivalent to $k_{1}-k_{2}>(1 / 4)^{t-1}$ $\times q^{0}\left(k_{1}-k_{2}\right)$ and $-\left(k_{1}-k_{2}\right)>(1 / 4)^{t-1} q^{0}\left(k_{1}-k_{2}\right)$. Thus $0>k_{1}-k_{2}$. But then the first inequality is contradicted since $1>1 / 5 \geqslant(1 / 4)^{t-1} q^{0}$. Of course, since $\liminf _{t \rightarrow \infty}\left\|p^{t}\right\|=0$, the equilibrium allocations are Pareto optimal and cannot be improved upon by the coalition of the whole. Thus the equilibrium allocations are in the core for every replication of the economy.

Q.E.D

The economy described in Proposition 3 does not contradict Esteban and Millan [10, Proposition 7] because the Gaussian curvature of each consumer's indifference curve evaluated at the equilibrium allocations is not bounded. ${ }^{10}$ But these preferences and endowments create such a strong inter-dependence on trade between generations that no coalition consisting of a subset of agents from two different generations can improve upon the equilibrium allocations. Of course, this particular example is not robust because it depends so crucially on the fact that goods within each period are perfect complements in each agent's preferences. Since the example consists of an economy with irreducible coalitions, the proof points towards an extension of Chae and Esteban [6, Theorem 1].

A monetary equilibrium is in the core if the gains from trade between generations are sufficiently important. ${ }^{11}$ Also, the example shows that the present price of the asset matters; the well-being of the initial generation is increasing in the present value of the stock of debt of which it is the beneficiary. Further, the example is robust with respect to the generational policy underlying the monetary equilibrium. In one sense, there is nothing special about the monetary economy described in the proof. Indeed, economies in which trade between generations is important and whose long-run real interest rates exceed the rate of growth of resources may be the norm, not the exception, in our world.

${ }^{10}$ Balasko and Shell [4] use an assumption bounding this curvature in characterizing Pareto optimal allocations.

${ }^{11}$ Fisher [11] has argued that gains from trade between agents in the same generation are also important in describing monetary equilibria supporting allocations in the core. 


\section{REFERENCES}

1. Alan J. Auerbach, J. Gokhale, and L. Kotlikoff, Generational accounts: A meaningful alternative to deficit accounting, in "Tax Policy and the Economy" (D. Bradford, Ed.), Vol. 5, Nat. Bur. of Econ. Res. and MIT Press, Cambridge, MA, 1991.

2. Yves Balasko, D. Cass, and K. Shell, Existence of competitive equilibrium in a general overlapping generations model, J. Econ. Theory 23 (1980), 307-322.

3. Yves Balasko and K. Shell, The overlapping generations model I: The case of pure exchange without money, J. Econ. Theory 23 (1980), 280-306.

4. Yves Balasko and K. Shell, The overlapping generations model II: The case of pure exchange with money, J. Econ. Theory 24 (1981), 112-142.

5. Yves Balasko and K. Shell, Lump-sum taxes and transfers: Public debt in the overlappinggenerations model, in "Equilibrium Analysis: Essays in Honor of Kenneth J. Arrow, Volume II” (W. P. Heller, R. M. Starr, and D. A. Starett, Eds.), Cambridge Univ. Press, Cambridge, UK, 1986.

6. Suchan Chae and J. Esteban, Core equivalence in an overlapping generations model, J. Econ. Theory 59 (1993), 417-425.

7. Gerard Debreu, The coefficient of resource utilization, Econometrica 19 (1951), 273-292.

8. Joan Esteban, The core in overlapping generations economies, J. Econ. Theory 39 (1986), $439-456$.

9. Joan Esteban, "The Social Viability of Money," Springer-Verlag, Berlin, 1991.

10. Joan Esteban and T. Millan, Competitive equilibria and the core of overlapping generations economies, J. Econ. Theory 50 (1990), 155-174.

11. Eric O'N. Fisher, On exchange rates and efficiency, Econ. Theory 7 (1996), 267-281.

12. David Gale, Pure exchange equilibrium of dynamic economic models, J. Econ. Theory 6 (1973), 12-36.

13. Douglas Gale, The core of an economy without trust, J. Econ. Theory 19 (1978), 456-491.

14. Ken Hendricks, K. Judd, and D. Kovenock, A note on the core of the overlapping generations model, Econ. Lett. 6 (1980), 95-97.

15. Dan Kovenock, A second note on the core of the overlapping generations model, Econ. Lett. 14 (1984), 101-106.

16. Karl Shell, Notes on the economics of infinity, J. Polit. Econ. 79 (1971), 1002-1011. 